\title{
Bounds for Lepton Flavor Violation and the Pseudoscalar Higgs in the General Two Higgs Doublet Model using $g-2$ muon factor
}

\author{
Rodolfo A. Diaz, R. Martinez, and J-Alexis Rodriguez \\ Departamento de Fisica, \\ Universidad Nacional de Colombia \\ Bogota, Colombia
}

\begin{abstract}
Current experimental data from the $g-2$ muon factor, seems to show the necessity of physics beyond the Standard Model (SM), since the difference between SM and experimental predictions is 2.6 $\sigma$. In the framework of the General Two Higgs Doublet Model (2HDM), we calculate the muon anomalous magnetic moment to get lower and upper bounds for the Flavour Changing (FC) Yukawa couplings in the leptonic sector. We also obtain lower bounds for the mass of the pseudoscalar Higgs $\left(m_{A^{0}}\right)$ as a function of the parameters of the model.
\end{abstract}


Current muon anomalous magnetic moment $a_{\mu}$ measurement has challenged Standard $\operatorname{Model}(\mathrm{SM})$ and seems to open a window for new physics. Due to the high precision in $a_{\mu}$ measurement, it gives very restrictive bounds on physics beyond the SM. Although $a_{e}$ measurement is about 350 more precise [1], $a_{\mu}$ is much more sensitive to New Physics since contributions to $a_{l}$ are usually proportional to $m_{l}^{2}$.

The most accurate measurement of $a_{\mu}$ hitherto, has been provided by the Brookhaven Alternating Gradient Syncrotron [2]. Their data have an error one third that of the combined previous data [3], ref [2] reports

$$
a_{\mu^{+}}=11659202(14)(6) \times 10^{-10} .
$$

On the other hand, SM predictions for $a_{\mu}$ has been estimated taking into account the contributions from QED, Hadronic loops and electroweak corrections. The final current result is [1], 2]

$$
a_{\mu}^{S M}=11659159.6(6.7) \times 10^{-10}
$$

Taking into account (1) and (2) is obtained

$$
\Delta a_{\mu}^{N P}=a_{\mu}^{\exp }-a_{\mu}^{S M}=42.6(16.5) \times 10^{-10}
$$

where $a_{\mu}^{\exp }$ is the world average experimental value. Consequently at $90 \%$ C.L.

$$
21.5 \times 10^{-10} \leq \Delta a_{\mu}^{N P} \leq 63.7 \times 10^{-10}
$$

$\Delta a_{\mu}^{N P}$ gives the room available for New Physics, so $a_{\mu}^{e x p}$ differs from $a_{\mu}^{S M}$ approximately in $2.6 \sigma$. Therefore, physics beyond the SM is needed to achieve an acceptable theoretical experimental agreement. The most studied contributions to $a_{\mu}$ has been carried out in the framework of radiative muon mass models as well as the Minimal Supersymmetric Standard Model (MSSM), $\mathrm{E}_{6}$ string-inspired models, and extensions of MSSM with an extra singlet [4].

Moreover, a very interesting suggestion to conciliate the new experimental data with theoretical predictions is to consider models that includes FCNC at tree level. Interactions involving FCNC are forbidden at tree level in the SM, but could be present at one loop level as in the case of $b \rightarrow s \gamma$ [5], $K^{0} \rightarrow \mu^{+} \mu^{-}$[6], $K^{0}-\bar{K}^{0}$ [7], $t \rightarrow c \gamma$ [8] etc. Many extensions of the SM permit FCNC at tree level. For example, the introduction of new representations of fermions different from doublets produce them by means of the Z-coupling [9]. Additionally, 
they are generated at tree level by adding a second doublet to the SM [10], such couplings can be gotten as well in SUSY theories without R-parity. Some other important new sources for FCNC might be provided by a muon collider, as the processes $\mu \mu \rightarrow \mu \tau(e \tau)$ mediated by Higgs exchange [11, [12], which produce Lepton Flavor Violation (LFV).

However, there are several mechanisms to avoid FCNC at tree level. Glashow and Weinberg [13] proposed a discrete symmetry to supress them in the Two Higgs Doublet Model (2HDM) which is the simplest one that exhibits these rare processes at tree level. There are two kinds of models which are phenomenologically plausible with the discrete symmetry imposed. In the model type I, one Higgs Doublet provides masses to the up-type and down-type quarks, simultaneously. In the model type II, one Higgs doublet gives masses to the up-type quarks and the other one to the down-type quarks. But the discrete symmetry [13] is not compulsory and both doublets may generate the masses of the quarks of up-type and down-type simultaneously, in such case we are in the model type III [14]. It has been used to search for FCNC at tree level [15], 116].

Recently, the 2HDM type III has been discussed and classified [17], depending on how the basis for the vacuum expectation values (VEV) are chosen and according to the way in which the flavor mixing matrices are rotated. In brief, the reference [17 shows that there are two types of rotations which generate four different lagrangians in the quark sector and two different ones in the leptonic sector. The well known 2HDM types I and II, could be generated from them in the limit in which the $\mathrm{FC}$ vertices vanish. It has been pointed out that the phenomenology of the $2 \mathrm{HDM}$ type III is highly sensitive to the rotation used for the mixing matrices.

In this paper, we calculate the contributions to $\Delta a_{\mu}$ coming from the $2 \mathrm{HDM}$, which includes FCNC at tree level. We will constrain the FC vertex involving the second and third charged leptonic sector by using the result for $\Delta a_{\mu}^{N P}$, equation (4). Additionally, we get lower bounds on the pseudoscalar Higgs mass by taking into account the lower experimental value of $\Delta a_{\mu}^{N P}$ at $90 \%$ C.L. by making reasonable assumptions on the $\mathrm{FC}$ vertex.

The Yukawa's Lagrangian for the 2HDM type III, is as follow

$$
\begin{aligned}
-£_{Y} & =\eta_{i j}^{U} \bar{Q}_{i L} \widetilde{\Phi}_{1} U_{j R}+\eta_{i j}^{D} \bar{Q}_{i L} \Phi_{1} D_{j R}+\eta_{i j}^{E} \bar{l}_{i L} \Phi_{1} E_{j R} \\
& +\xi_{i j}^{U} \bar{Q}_{i L} \widetilde{\Phi}_{2} U_{j R}+\xi_{i j}^{D} \bar{Q}_{i L} \Phi_{2} D_{j R}+\xi_{i j}^{E} \bar{l}_{i L} \Phi_{2} E_{j R}+\text { h.c. }
\end{aligned}
$$

where $\Phi_{1,2}$ are the Higgs doublets, $\eta_{i j}$ and $\xi_{i j}$ are non-diagonal $3 \times 3$ matrices and $i, j$ are 
family indices. In this work, we are interested only in neutral currents in the leptonic sector. We also consider a CP-conserving model in which both Higgs doublets acquire a VEV,

$$
\left\langle\Phi_{1}\right\rangle_{0}=\left(\begin{array}{c}
0 \\
v_{1} / \sqrt{2}
\end{array}\right),\left\langle\Phi_{2}\right\rangle_{0}=\left(\begin{array}{c}
0 \\
v_{2} / \sqrt{2}
\end{array}\right) \text {. }
$$

The neutral mass eigenstates are given by 18

$$
\begin{aligned}
\left(\begin{array}{c}
G_{Z}^{0} \\
A^{0}
\end{array}\right) & =\left(\begin{array}{cc}
\cos \beta & \sin \beta \\
-\sin \beta & \cos \beta
\end{array}\right)\left(\begin{array}{c}
\sqrt{2} \operatorname{Im} \phi_{1}^{0} \\
\sqrt{2} \operatorname{Im} \phi_{2}^{0}
\end{array}\right), \\
\left(\begin{array}{c}
H^{0} \\
h^{0}
\end{array}\right) & =\left(\begin{array}{cc}
\cos \alpha & \sin \alpha \\
-\sin \alpha & \cos \alpha
\end{array}\right)\left(\begin{array}{c}
\sqrt{2} \operatorname{Re} \phi_{1}^{0}-v_{1} \\
\sqrt{2} \operatorname{Re} \phi_{2}^{0}-v_{2}
\end{array}\right)
\end{aligned}
$$

where $\tan \beta=v_{2} / v_{1}$ and $\alpha$ is the mixing angle of the CP-even neutral Higgs sector. $G_{Z}$ is the would-be Goldstone boson of $Z$ and $A^{0}$ is the CP-odd neutral Higgs.

Now, to convert the Lagrangian (5) into mass eigenstates we make the unitary transformations

$$
E_{L, R}=\left(V_{L, R}\right) E_{L, R}^{0}
$$

from which we obtain the mass matrices

$$
M_{E}^{\text {diag }}=V_{L}\left[\frac{v_{1}}{\sqrt{2}} \eta^{E, 0}+\frac{v_{2}}{\sqrt{2}} \xi^{E, 0}\right] V_{R}^{\dagger},
$$

where $M_{E}^{\text {diag }}$ is the diagonal mass matrix for the three lepton families. From (9) we can solve for $\xi^{E, 0}$ obtaining

$$
\xi^{E, 0}=\frac{\sqrt{2}}{v_{2}} V_{L}^{\dagger} M_{E}^{d i a g} V_{R}-\frac{v_{1}}{v_{2}} \eta^{E, 0} .
$$

which we call a rotation of type I. Replacing it into (5), the expanded Lagrangian for the neutral leptonic sector is

$$
\begin{aligned}
-£_{Y(E)}^{(I)} & =\frac{g}{2 M_{W} \sin \beta} \bar{E} M_{E}^{\text {diag }} E\left(\sin \alpha H^{0}+\cos \alpha h^{0}\right) \\
& +\frac{i g}{2 M_{W}} \bar{E} M_{E}^{\text {diag }} \gamma_{5} E G^{0}+\frac{i g \cot \beta}{2 M_{W}} \bar{E} M_{E}^{\text {diag }} \gamma_{5} E A^{0} \\
& -\frac{1}{\sqrt{2} \sin \beta} \bar{E} \eta^{E} E\left[\sin (\alpha-\beta) H^{0}+\cos (\alpha-\beta) h^{0}\right] \\
& -\frac{i}{\sqrt{2} \sin \beta} \bar{E} \eta^{E} \gamma_{5} E A^{0}+\text { h.c. }
\end{aligned}
$$

where the superindex $(I)$ refers to the rotation type I. It is easy to check that Lagrangian (11) is just the one in the 2HDM type I [18], plus some FC interactions. Therefore, we 
obtain the lagrangian of the 2HDM type I from eq (11) by setting $\eta^{E}=0$. In this case it is clear that when $\tan \beta \rightarrow 0$ then $\eta^{E}$ should go to zero, in order to have a finite contribution for FCNC at tree level.

On the other hand, from (9) we can also solve for $\eta^{E, 0}$ instead of $\xi^{E, 0}$, to get

$$
\eta^{E, 0}=\frac{\sqrt{2}}{v_{1}} V_{L}^{\dagger} M_{E}^{d i a g} V_{R}-\frac{v_{2}}{v_{1}} \xi^{E, 0}
$$

which we call a rotation of type II. Replacing it into (5) the expanded Lagrangian for the neutral leptonic sector is

$$
\begin{aligned}
-£_{Y(E)}^{(I I)} & =\frac{g}{2 M_{W} \cos \beta} \bar{E} M_{E}^{\text {diag }} E\left(\cos \alpha H^{0}-\sin \alpha h^{0}\right) \\
& +\frac{i g}{2 M_{W}} \bar{E} M_{E}^{\text {diag }} \gamma_{5} E G^{0}-\frac{i g \tan \beta}{2 M_{W}} \bar{E} M_{E}^{\text {diag }} \gamma_{5} E A^{0} \\
& +\frac{1}{\sqrt{2} \cos \beta} \bar{E} \xi^{E} E\left[\sin (\alpha-\beta) H^{0}+\cos (\alpha-\beta) h^{0}\right] \\
& +\frac{i}{\sqrt{2} \cos \beta} \bar{E} \xi^{E} \gamma_{5} E A^{0}+\text { h.c. }
\end{aligned}
$$

The Lagrangian (13) coincides with the one of the 2HDM type II [18, plus some FC interactions. So, the lagrangian of the $2 \mathrm{HDM}$ type II is obtained setting $\xi^{E}=0$. In this case it is clear that when $\tan \beta \rightarrow \infty$ then $\xi^{E}$ should go to zero, in order to have a finite contribution for FCNC at tree level.

In the present report, we calculate $a_{\mu}$ in the 2HDM with FC interactions. If we neglect the muon mass, the contribution at one loop from all Higgses is given by

$$
\Delta a_{\mu}^{N P}=\frac{m_{\mu} m_{l}}{16 \pi^{2}} \sum_{i} F\left(m_{H_{i}}, m_{l}\right) a_{i}^{2}
$$

where

$$
F\left(m_{H_{i}}, m_{l}\right)=\frac{\widehat{m}_{H_{i}}^{2}\left(\widehat{m}_{H_{i}}^{2}-4\right)+\left[3+2 \ln \left(\widehat{m}_{H_{i}}^{2}\right)\right]}{m_{H_{i}}^{2}\left(1-\widehat{m}_{H_{i}}^{2}\right)^{3}}
$$

with $\widehat{m}_{H_{i}}=m_{l} / m_{H_{i}}$ and $m_{l}$ is the mass of the lepton running into the loop. The sum is over the index $i=m_{h^{0}}, m_{H^{0}}, m_{A^{0}}$. The coefficients $a_{i}$ are the Feynman rules for FC couplings involved.

If we take into account the experimental data (4), we get some lower and upper bounds on the mixing vertex $\eta(\xi)_{\mu \tau}$ for the rotations of type I (II). In figure 1, we display lower and upper bounds for the FC vertices as a function of the $\tan \beta$ for both types of rotations with $m_{h^{0}}=m_{H^{0}}=150 \mathrm{GeV}$ and $m_{A^{0}} \rightarrow \infty$. For $\tan \beta=1$ the behaviour of the bounds 
for both rotations is the same. In the first case, rotation type I, the allowed region for $\eta_{\mu \tau}$ is between $0.07 \leq \eta_{\mu \tau} \leq 0.13$ for large values of $\tan \beta$. Meanwhile, for rotation type II, the allowed region for small $\tan \beta$ is the same. From Lagrangian (11), rotation type I, we can see that when $\tan \beta \rightarrow 0, \eta_{\mu \tau}$ should go to zero as well to mantain a finite contribution to $\Delta a_{\mu}$. This behaviour can be seen from figure 1. For rotation type II is similar to the former but in the limit $\tan \beta \rightarrow \infty$.

In figure 2 , we show lower and upper bounds for the FC vertex as a function of $m_{H^{0}}$ for rotation of type II when $m_{h^{0}}=m_{H^{0}}$ and $m_{A^{0}} \rightarrow \infty$. We see that the smaller value of $\xi_{\mu \tau}$ the larger value for $\tan \beta$. We only consider the case of rotation type II because there is a complementary behaviour as could be seen in figure 1 .

Observe that according to the Feynman rules from (11) and (13), the scalar (pseudoscalar) contribution to $\Delta a_{\mu}^{N P}$ eq. (15) is positive (negative). Such fact permits us to impose lower bounds on the pseudoscalar Higgs mass, by using the lower limit in eq. (四). According to this equation the room for new physics from $g-2$ muon factor is positive definite, and it is a new feature from most updated results [2].

Now, to take into account the experimental value (ब), we should make a supposition about the value of the $\mathrm{FC}$ vertex. A reasonable assumption consists of taking the geometric average of the Yukawa couplings [19] i.e. $\eta(\xi)_{\mu \tau} \approx 2.5 \times 10^{-3}$. Additionally, we shall use also the values $\eta(\xi)_{\mu \tau} \approx 2.5 \times 10^{-2}$ and $\eta(\xi)_{\mu \tau} \approx 2.5 \times 10^{-4}$ which are one order of magnitude larger and smaller than the former. Using these suppositions and the experimental value (困) we get restrictions for $m_{A^{0}}$ and they are plotted in figures (3)-(5).

Figure 3 displays $m_{A^{0}}$ vs $\tan \beta$ using rotation type II with the three values of $\xi_{\mu \tau}$ mentioned above and setting $m_{h^{0}}=m_{H^{0}}$ with $m_{h^{0}}=110,300 \mathrm{GeV}$. It could be seen that in the limit of large $\tan \beta$, the lower limit reduces to $m_{A^{0}} \approx m_{h^{0}}$. The same behavior can be seen in rotation type I but the bound $m_{A^{0}} \approx m_{h^{0}}$ is gotten in the limit of small $\tan \beta$. We see that the smaller value of $\xi_{\mu \tau}$ the stronger lower limit for $m_{A^{0}}$.

Figure 4 shows $m_{A^{0}}$ vs $m_{h^{0}}$ with $\xi_{\mu \tau}=2.5 \times 10^{-3}, 2.5 \times 10^{-2}$ and setting $\tan \beta=1, \alpha=$ $\pi / 2$ and using $m_{h^{0}}=m_{H^{0}}=110 \mathrm{GeV}$. With this settings, the value $\xi_{\mu \tau}=2.5 \times 10^{-4}$ is excluded. Using such specific arrangements, the bounds are identical in both types of rotations.

In figure 5 we suppose that $m_{h^{0}} \neq m_{H^{0}}$ and the bounds are function of $\sin \alpha$. The above figure shows the sensitivity of lower bounds on $m_{A^{0}}$ with the mixing angle $\alpha$, for rotation 
type II. The value $\xi_{\mu \tau}=2.5 \times 10^{-4}$ is excluded again. The constraints are very sensitive for $\xi_{\mu \tau}=2.5 \times 10^{-3}$ respect to $\sin \alpha$ but rather insensitive for $\xi_{\mu \tau}=2.5 \times 10^{-2}$. The figure below shows $m_{A^{0}}$ vs $\tan \beta$ for $m_{h^{0}}=110 \mathrm{GeV}, m_{H^{0}}=300 \mathrm{GeV}, \alpha=\pi / 6$, for rotation type II and using the same three values of $\xi_{\mu \tau}$. The $m_{A^{0}}$ lower asymptotic limit for large $\tan \beta$ is approximately $m_{h^{0}}$.

In conclusion, we have found lower and upper bounds for the FC vertex $\eta(\xi)_{\mu \tau}$ in the context of the general 2HDM by using the allowed range for $\Delta a_{\mu}^{N P}$ at $90 \%$ C.L. and utilizing several sets of values for parameters of the model. Additionally, in the limit $m_{A^{0}} \rightarrow \infty$ we get that for small (large) values of $\tan \beta$ the allowed range for the FC vertex $\eta_{\mu \tau}\left(\xi_{\mu \tau}\right)$ becomes narrower, and both upper and lower bounds go to zero in the rotation of type I (II).

On the other hand, we have gotten lower bounds on the pseudoscalar Higgs mass of the 2HDM coming from the $g-2$ muon factor, by using the experimental value of $\Delta a_{\mu}^{N P}$ and making reasonable assumptions on the FC vertex $\eta(\xi)_{\mu \tau}$. Specifically, we have taken for $\eta(\xi)_{\mu \tau}$ the geometric average of the Yukawa couplings, and we also utilized values one order of magnitude larger and one smaller. Taking these three values for the FC vertex we find that the smaller value for $\eta(\xi)_{\mu \tau}$ the more stringent lower bounds for $m_{A^{0}}$. Additionally, assuming $m_{H^{0}}=m_{h^{0}}$, we show that in the limit of small (large) $\tan \beta$ the lower bound of $m_{A^{0}}$ becomes merely $m_{A^{0}} \approx m_{h^{0}}$ for rotation of type I (II). In the case of different scalar masses, there is still a lower asymptotic limit for $m_{A^{0}}$. Notwithstanding, these lower constraints on $m_{A^{0}}$ should be consider carefully, since for $\eta(\xi)_{\mu \tau}$ we can only make reasonable estimations but they are unknown so far.

This work was supported by COLCIENCIAS, DIB and DINAIN.

[1] A. Czarnecki and W. Marciano, hep-ph/0102122.

[2] H.N. Brown et. al.; hep-ex/0102017

[3] C. Caso et.al. (Particle Data Group), Eur. Phys. J. C3, 1 (1998).

[4] J. A. Grifols and A. Mendez, Phys. Rev. D26, 1809 (1989); M. Frank and C. S. Kalman, Phys. Rev. D38, 1469 (1988); J. A. Grifols, J. Sola and A. Mendez, Phys. Rev. Let. 57, 2348 (1986); M. Krawczyk and J. Zochowski, Phys. Rev. D55, 6968 (1997); U. Chattopadhyay and P. Nath, hep-ph/0102157; D. Choudhry, B. Mukhopadhyaya and S. Rakshit, hep-ph/0102199. 
[5] M. Ciuchini, et. al., Phys. Lett. B 316, 127 (1993); Nucl. Phys. B 421, 41 (1994); S. Bertolini, F. Borzumati, A. Masiero and G. Ridolfi, Nucl. Phys. B 353591 (1991).

[6] H. Stern and M. K. Gaillard, Ann. Phys. 76, 580 (1973); C. S. Kim, J. L. Rosner and C. P Yuan, Phys. Rev. D 42, 96 (1990).

[7] T. Imami and C. S. Lim, Prog. The. Phys. 65, 297 (1981).

[8] J. L. Diaz-Cruz, et. al., Phys. Rev. D 41, 891 (1990); G. Eilam, J. Hewett and A. Soni, Phys. Rev D 44, 1473 (1991); G. Couture, C. Hamzanoi and H. Konig, Phys. Rev. D 49, 293 (1995).

[9] J. L. Hewett and T. Rizzo, Phys. Rep. 183, 193 (1989); G. Baremboim, et. al, Phys. Lett. B 422, 277 (1998); V. Barger, M. Berger and R. Phillips, Phys. Rev. D 52, 1663 (1995); R. Martinez, J.-Alexis Rodriguez and M. Vargas, Phys. Rev. D 60, 077504 (1999); F. del Aguila, J. Aguilar Saavedra and R. Miquel, Phys. Rev. Lett. 82, 1628 (1999).

[10] J. Liu and L. Wolfenstein, Nucl. Phys. B 289, 1 (1987).

[11] Workshop on Physics at the First Muon Collider and at the Front End of the Muon Collider, ed. S. Geer and R. Raja (AIP Publishing, Batavia Ill 1997).

[12] Marc Sher, hep-ph/0006159v3.

[13] S. Glashow and S. Weinberg, Phys. Rev. D 15, 1958 (1977).

[14] W.S. Hou, Phys. Lett B 296, 179 (1992); D. Cahng, W. S. Hou and W. Y. Keung, Phys. Rev. D 48, 217 (1993); J.L. Diaz-Cruz, J.J. Godina and G. López Castro, Phys. Lett B 301 (93) 405 .

[15] D. Atwood, L. Reina and A. Soni, Phys. Rev. D 53, 1199 (1996); Phys. Rev. D 54, 3296 (1996); Phys. Rev. Lett. 75, 3800 (1993); D. Atwood, L. Reina and A. Soni, Phys. Rev. D 55, 3156 (1997); G. Cvetic, S. S. Hwang and C. S. Kim., Phys. Rev. D 58, 116003 (1998).

[16] Marc Sher and Yao Yuan, Phys. Rev. D 44, 1461 (1991).

[17] Rodolfo A. Diaz, R. Martinez and J.-Alexis Rodriguez, hep-ph/0010149. To appear in Phys. Rev. D.

[18] For a review see J. Gunion, H. Haber, G. Kane and S. Dawson, The Higgs Hunter's Guide, (Addison-Wesley, New York, 1990).

[19] T.P. Cheng and M. Sher, Phys. Rev. D 35, 3490 (1987) 

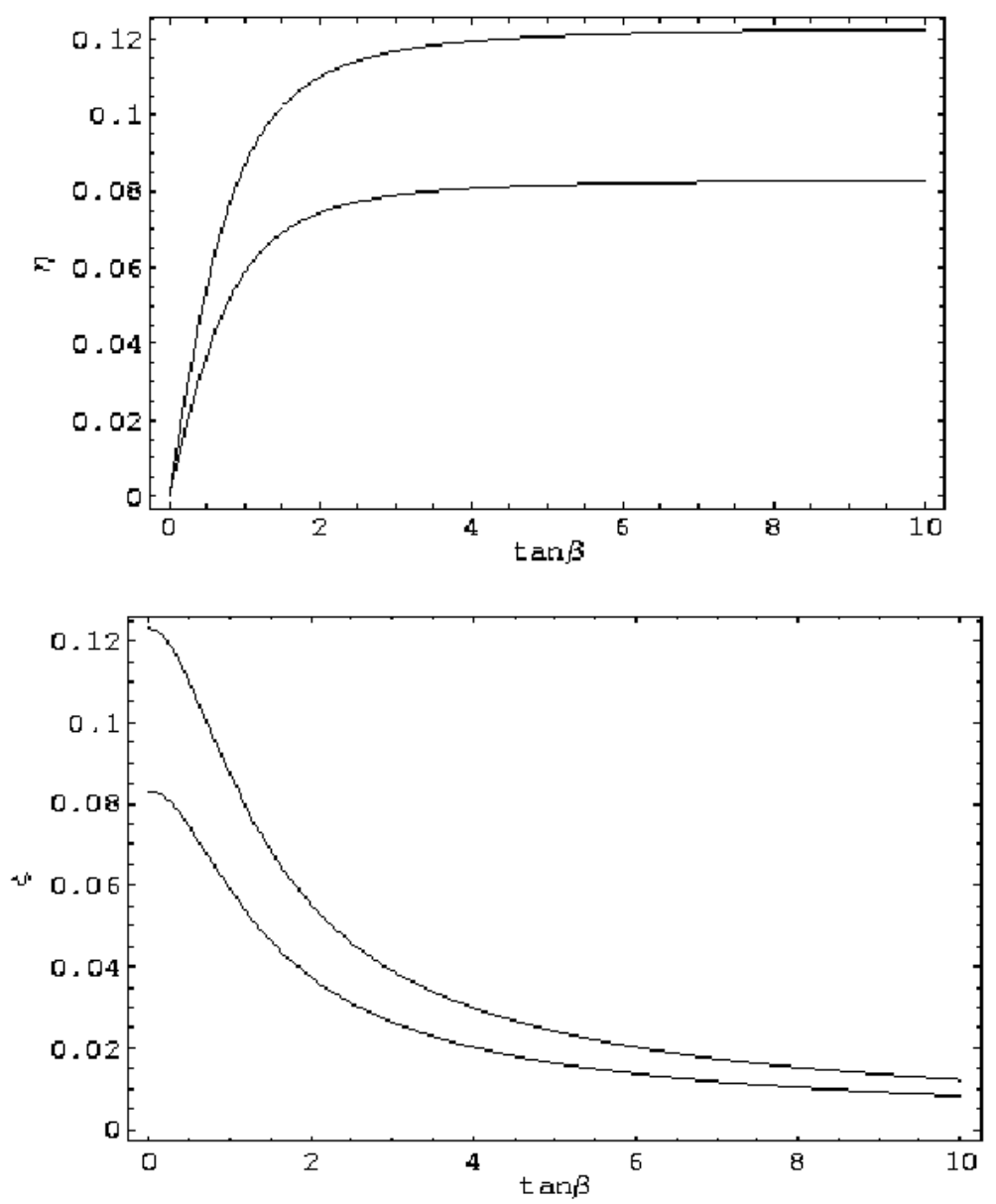

FIG. 1: Lower and upper bounds for $\eta_{\mu \tau}\left(\xi_{\mu \tau}\right)$ vs $\tan \beta$, for rotations I and II using $m_{h^{0}}=m_{H^{0}}=$ $150 \mathrm{GeV}$ and $m_{A^{0}} \rightarrow \infty$. 


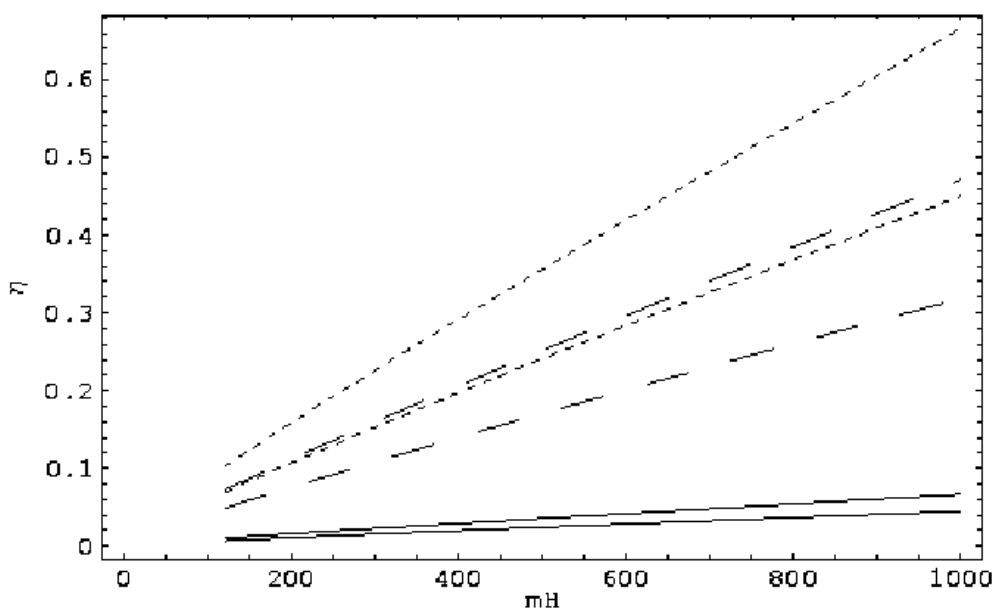

FIG. 2: Figure 2. Lower and upper bounds for $\eta_{\mu \tau}\left(\xi_{\mu \tau}\right)$ vs $m_{H^{0}}$, for rotation of type I, taking $m_{h^{0}}=m_{H^{0}}$ and $m_{A^{0}} \rightarrow \infty$, the pair of short dashed lines correspond to $\tan \beta=30$, the long dashed lines are for $\tan \beta=1$, and the continuous lines are for $\tan \beta=0.1$. 


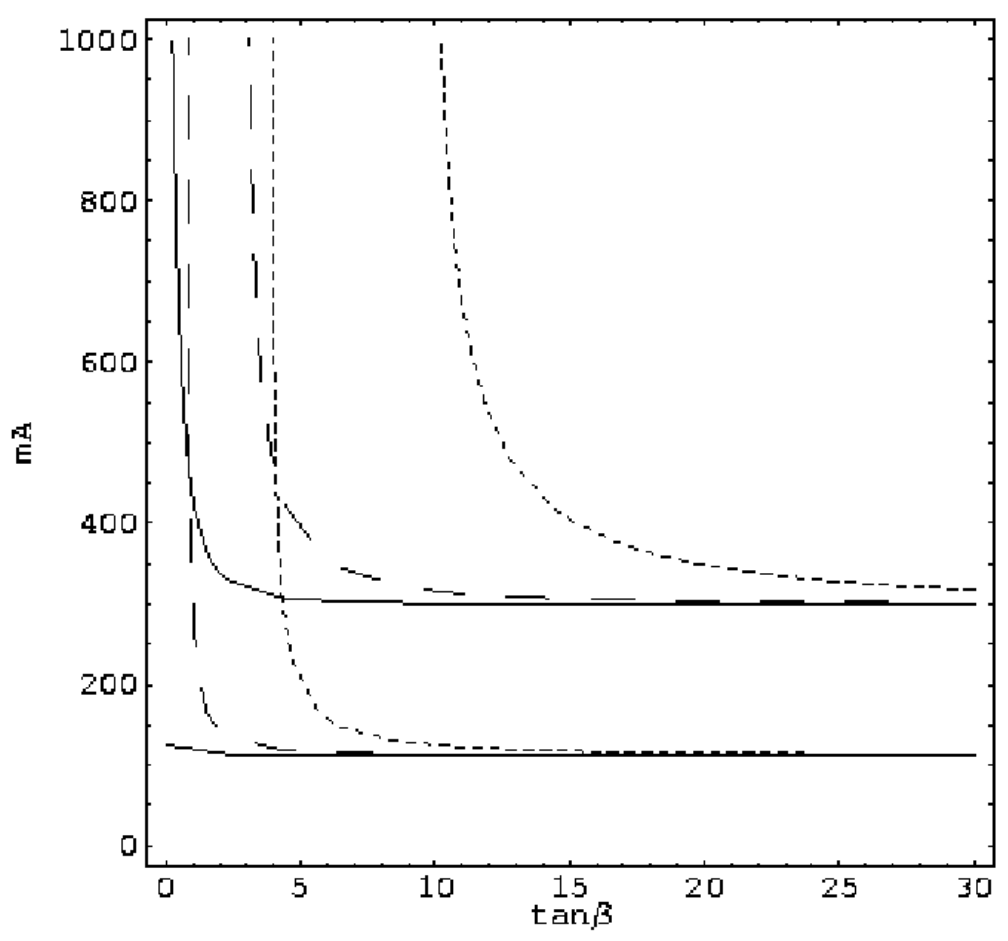

FIG. 3: Contour plot of $m_{A^{0}}$ vs $\tan \beta$ using rotation type II and assuming $m_{h^{0}}=m_{H^{0}}$. Short dashed lines correspond to $\xi_{\mu \tau}=2.5 \times 10^{-4}$ for $m_{H^{0}}=110 \mathrm{GeV}$ (below) and $m_{H^{0}}=300 \mathrm{GeV}$ (above). Long dashed lines correspond to $\xi_{\mu \tau}=2.5 \times 10^{-3}$ for $m_{H^{0}}=110 \mathrm{GeV}$ (below) and $m_{H^{0}}=300 \mathrm{GeV}$ (above). Finally, solid lines correspond to $\xi_{\mu \tau}=2.5 \times 10^{-3}$ for $m_{H^{0}}=110 \mathrm{GeV}$ (below) and $m_{H^{0}}=300 \mathrm{GeV}$ (above). 


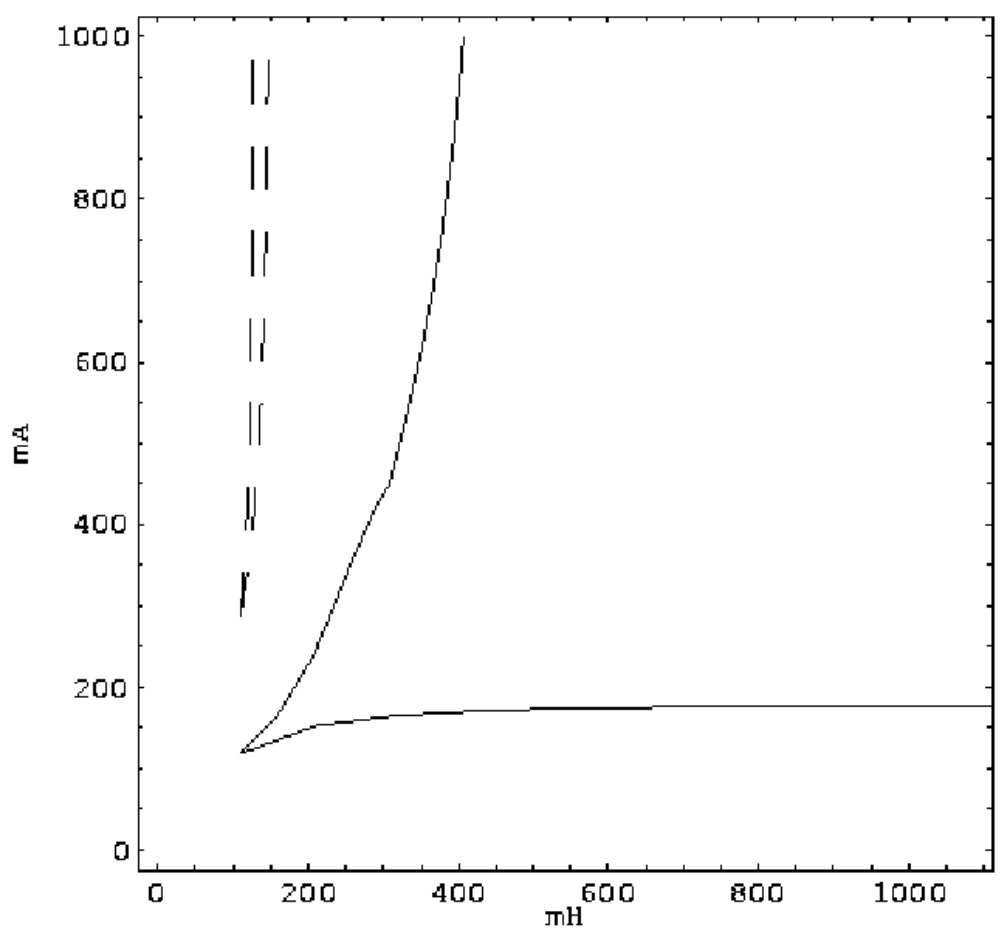

FIG. 4: Contour plot of $m_{A^{0}}$ vs $m_{h^{0}}$ setting $\tan \beta=1, \alpha=\pi / 2$. Long dashed lines correspond to $\xi_{\mu \tau}=2.5 \times 10^{-3}$ for $m_{H^{0}}=110 \mathrm{GeV}$ (below) and $m_{h^{0}}=m_{H^{0}}$ (above). Solid lines correspond to $\xi_{\mu \tau}=2.5 \times 10^{-2}$ for $m_{h^{0}}=110 \mathrm{GeV}$ (below) and $m_{h^{0}}=m_{H^{0}}$ (above). 

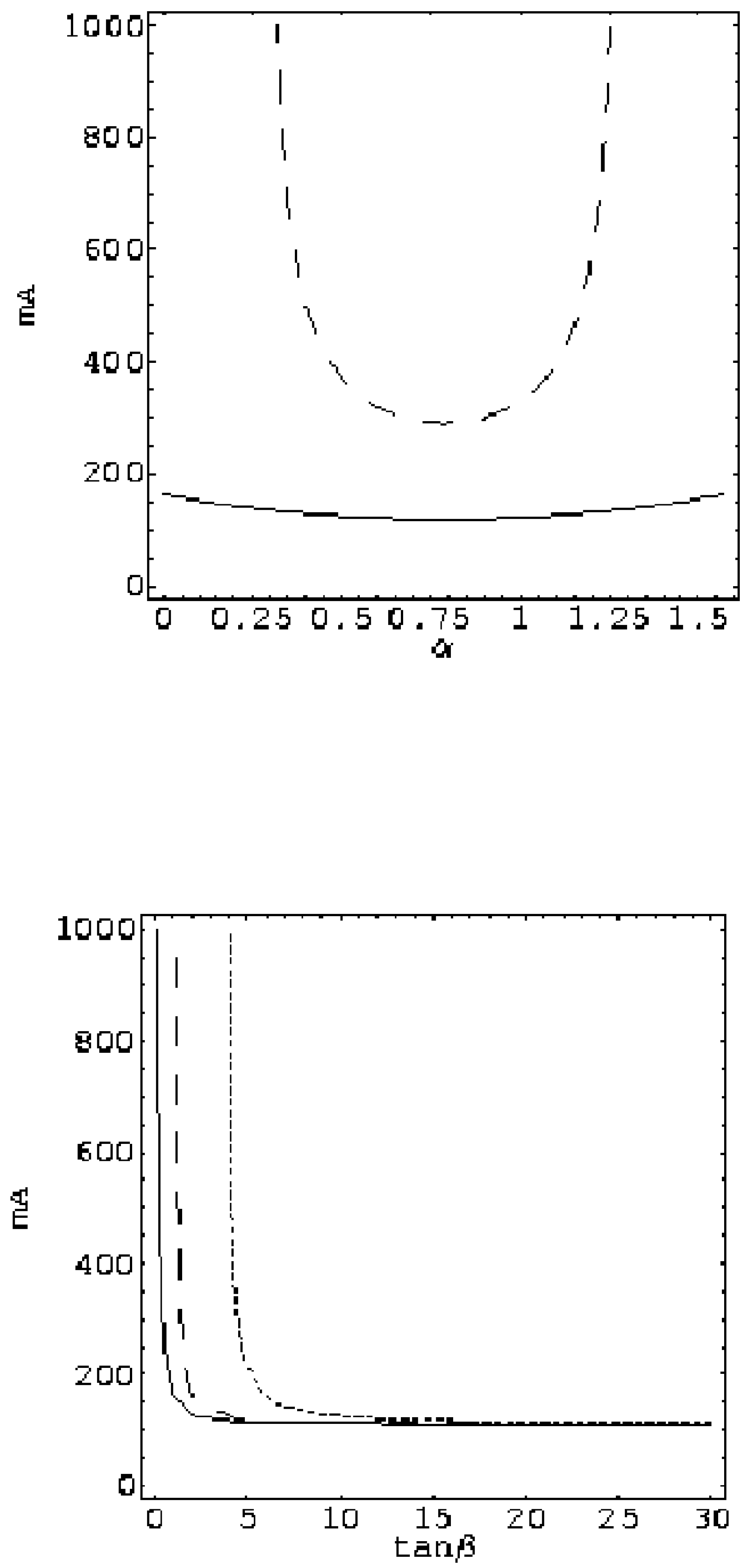

FIG. 5: (top) Contour plot of $m_{A^{0}}$ vs $\alpha$, for rotation type II. Dashed line correspond to $\xi_{\mu \tau}=$ $2.5 \times 10^{-3}$, solid line correspond to $\xi_{\mu \tau}=2.5 \times 10^{-2}$. (bottom) Contour plot of $m_{A^{0}}$ vs $\tan \beta$ for $m_{H^{0}}=300 \mathrm{GeV}, m_{h^{0}}=110 \mathrm{GeV}, \alpha=\pi / 6$, anłlfor rotation type II. Short dashed line correspond to $\xi_{\mu \tau}=2.5 \times 10^{-4}$, long dashed line correspond to $\xi_{\mu \tau}=2.5 \times 10^{-3}$, and solid line correspond to 